\title{
Chlamydia pneumoniae infection in adult patients with persistent cough
}

\author{
Naoyuki Miyashita, Hiroshi Fukano, Koichiro Yoshida, Yoshihito Niki and \\ Toshiharu Matsushima
}

\author{
Correspondence \\ Naoyuki Miyashita \\ nao@med.kawasaki-m.ac.jp
}

Received 6 June 2002

Accepted 31 October 2002

\author{
Division of Respiratory Diseases, Department of Medicine, Kawasaki Medical School, 577 \\ Matsushima, Kurashiki, Okayama 701-0192, Japan
}

\begin{abstract}
Chlamydia pneumoniae is a frequent causative agent of acute respiratory disease. To assess whether C. pneumoniae plays a role in persistent cough, the prevalence of C. pneumoniae infection in adult patients with persistent cough was investigated. Nasopharyngeal swabs and serology samples from 366 adult patients with a persistent cough lasting in excess of 2 weeks and 106 control subjects were analysed for bacterial isolation and by PCR. C. pneumoniae was isolated from two patients and from none of the controls and was detected by PCR in 20 patients and one control. Serological evidence of acute C. pneumoniae infection was present in 24 patients but in none of the controls. Of these 20 patients who were positive by culture and/or PCR, three were still positive by PCR after 2 weeks of treatment with clarithromycin and symptoms either continued or relapsed. However, when patients were treated with clarithromycin for 5-6 weeks, their symptoms disappeared completely and the results of their cultures and/or PCR for C. pneumoniae became negative. These data suggest that $C$. pneumoniae infection may cause persistent cough in adults. Furthermore, these data also indicate that it may be necessary to eradicate the organism when $C$. pneumoniae is detected by culture and/or PCR in patients with persistent cough.
\end{abstract}

\section{INTRODUCTION}

Chlamydia pneumoniae has been shown to cause both epidemic and endemic respiratory tract infections in many areas of the world (Grayston et al., 1990; Kuo et al., 1995). It is a common cause of lower and upper respiratory illnesses, including pneumonia, bronchitis, pharyngitis and sinusitis, in both children and adults (Grayston et al., 1990; Kuo et al., 1995). Furthermore, C. pneumoniae infection has been found to establish persistent infections (Hammerschlag et al., 1992; Miyashita et al., 1996a; Ekman et al., 1993; Falck et al., 1996) and it has been reported that symptoms associated with C. pneumoniae infection may persist for a long time (Hammerschlag et al., 1992; Miyashita et al., 1996a; Ekman et al., 1993; Falck et al., 1996, 1997; Normann et al., 1998). Clinical symptoms of $C$. pneumoniae infections are nonspecific but a cough is the most common symptom and this is often prolonged (Kuo et al., 1995; Hammerschlag et al., 1992; Miyashita et al., 1996a; Soda et al., 1997). In a previous study, we encountered many cases of $C$. pneumoniae respiratory tract infection in junior high school students, and in related family members, during an outbreak of C. pneumoniae at their school (Soda et al., 1997). During this outbreak, many students and their family members had a persistent cough that lasted for more than 2 weeks.

Patients with persistent cough frequently make multiple visits to a physician, are given several courses of antibiotics and miss time from work. If their symptoms do not improve, they may finally visit a respiratory physician. However, the role of $C$. pneumoniae infection in adult patients with persistent cough has not been well evaluated, especially in Asia. In this study, we investigated prospectively an association between persistent cough and C. pneumoniae infection by isolation in cell culture, by PCR from nasopharyngeal swabs and by serology.

\section{METHODS}

Study population. The patients studied were 366 adults ( 133 males and 233 females) between the ages of 17 and 61 years (mean age, $36 \cdot 1$ years). All patients had a persistent cough lasting more than 2 weeks; this timeframe has been used previously as the expected time-frame of a prolonged C. pneumoniae cough (Wright et al., 1997). These patients were seen at the Kawasaki Medical School Hospital and Kurashiki Daiichi Hospital between April 1998 and December 2001. Patients with a cough lasting for more than 3 months, taking angiotensin-converting enzyme inhibitors or with known or suspected immunodeficiency, lung cancer, pneumonia, tuberculosis, chronic obstructive pulmonary diseases, bronchiectasis, interstitial lung diseases, chronic sinusitis, gastroesophageal reflux, allergic rhinitis or asthma were excluded. Control subjects without complaints of a cough were selected from healthy blood donors during the study period and were matched for age, sex and smoking status. The criteria for inclusion were no signs or symptoms of acute respiratory illness during the preceding 3 months. Informed consent was obtained from all subjects. 
Culture and PCR. Nasopharyngeal swab specimens were obtained from all subjects for isolation in cell culture and for PCR. Swab specimens were placed in a sucrose/phosphate/glutamate (SPG) transport medium. Each swab specimen in SPG medium was sonicated and centrifuged briefly at $900 \mathrm{~g}$ for $10 \mathrm{~min}$. Supernatants were inoculated onto confluent monolayers of HEp-2 cells grown on round coverslips ( $14 \mathrm{~mm}$ in diameter) set in 24 -well plastic cell culture plates. Plates were centrifuged at $1200 \mathrm{~g}$ for $60 \mathrm{~min}$ at room temperature. Next, $1 \mathrm{ml}$ of Eagle's minimal essential medium (Nissui Pharmaceuticals) supplemented with $10 \%$ heat-inactivated foetal calf serum (Gibco-BRL) and cycloheximide (final concentration of $1 \mu \mathrm{g} \mathrm{ml}^{-1}$; Nakarai Tesque) was applied to each well. Plates were incubated in $5 \% \mathrm{CO}_{2}$ at $35^{\circ} \mathrm{C}$ for $72 \mathrm{~h}$; all specimens were passaged twice (Miyashita et al., 1996b, 2001c). Following incubation, a genus-specific, FITC-conjugated monoclonal antibody (mAb) (Chlamydia FA Seiken; Denka Seiken) and C. pneumoniae species-specific mAbs were used to stain inclusions (Miyashita et al., 1996b). Inclusions were observed under a Nikon epifluorescence microscope at a magnification of $\times 200$ or $\times 400$.

C. pneumoniae-specific primers used for PCR were based on the DNA sequence within the $53 \mathrm{kDa}$ protein gene established by our laboratory (Miyashita et al., 1996b). This assay was performed as described previously and was carried out without prior knowledge of the culture results. The cell culture-grown C. pneumoniae strain KKp-15 was used as the positive control (Miyashita et al., 1994). SPG transport medium was used as the negative control in every run. After electrophoresis of PCR products on a $1.5 \%$ agarose gel at $100 \mathrm{~V}$, bands were visualized by staining with ethidium bromide. The appearance of a 499 bp PCR amplification product was taken as positive. If available, follow-up nasopharyngeal swab specimens were obtained from subjects with positive findings by culture and/or PCR.

Serology. Paired serum samples were obtained from all subjects at intervals of at least 4 weeks and stored at $-70{ }^{\circ} \mathrm{C}$ until testing. The microimmunofluorescence (MIF) test was used for the titration of IgG and IgM antibodies against C. pneumoniae (Grayston et al., 1990; Kuo et al., 1995) using formalin-treated elementary bodies of $C$. pneumoniae TW-183 (purchased from the Washington Research Foundation, Seattle, WA, USA) and KKpn-15 strains as antigens (Miyashita et al., 1994). Sera with IgM against C. pneumoniae were retested after absorption with goat anti-human IgG antibody reagent (Gullsorb) to exclude false-positive reactions. An acute infection was defined as one that gave IgM titres $\geqslant 1: 16$ or a fourfold increase in IgG or IgM titres.

Complement fixation and passive agglutinin tests were used to detect antibodies to Mycoplasma pneumoniae. A fourfold increase in antibody titres was taken as an indication of acute infection. Pertussis toxin (PT) and filamentous haemagglutinin (FHA) tests were performed to detect antibodies to Bordetella pertussis using ELISAs. Serological diagnosis of $B$. pertussis was based either on a fourfold increase in PT and/or FHA titre or a single PT or FHA result that was more than 2 SD greater than the geometric mean of the control group (Wright et al., 1995).

Statistical analysis. Statistical analysis was done using the Fisher Exact test and the Chi-squared test. A mean age comparison was carried out using Student's $t$-test.

\section{RESULTS}

Study population demographics are shown in Table 1 . No significant differences were observed between patients and controls with regard to age, sex or smoking status.

C. pneumoniae was detected by isolation in two patients $(0.5 \%)$ but in none of the controls $(P=0.902)$ and by PCR in 20 patients $(5 \cdot 5 \%)$ and in one control $(0 \cdot 9 \%)(P=0 \cdot 048)$. Both culture-positive specimens were also PCR positive. Serological evidence of acute $C$. pneumoniae infection was present in 24 patients $(6.5 \%)$ but in none of the controls $(P=0 \cdot 004)$. Thirteen cases were positive by both PCR and serology and 18 cases were positive with discrepancies in the PCR and serology results. Eleven cases were PCR negative and serology positive and seven cases were PCR positive and serology negative.

Other respiratory tract pathogens, including $B$. pertussis and M. pneumoniae, were identified in $68(18.5 \%)$ and $4(1 \cdot 1 \%)$ patients, respectively, by serology. Dual infections of $C$.

Table 1. Study population demographics

\begin{tabular}{|lccc|}
\hline Characteristic & Patient & Control & P value \\
\hline Number of subjects & 366 & 106 & \\
Age (years; mean \pm SD) & $36 \cdot 1 \pm 10 \cdot 6$ & $36 \cdot 0 \pm 9 \cdot 6$ & $0 \cdot 802$ \\
Age range (years) & $17-61$ & $19-60$ & \\
Sex (males; \%) & $133(36 \cdot 3)$ & $38(35 \cdot 8)$ & 0.926 \\
Smoking status (\%) & & & \\
Current & $134(36 \cdot 6)$ & $42(39 \cdot 6)$ & \\
Past & $106(29 \cdot 0)$ & $29(27 \cdot 4)$ & \\
Never & $126(34 \cdot 4)$ & $35(33 \cdot 0)$ & $0 \cdot 788^{\star}$ \\
C. pneumoniae infection (\%) & & & \\
Culture & $2(0 \cdot 5)$ & 0 & $1 \cdot 000$ \\
PCR & $20(5 \cdot 5)$ & $1(0 \cdot 9)$ & $0 \cdot 048$ \\
Serology $\dagger$ & $24(6 \cdot 5)$ & 0 & $0 \cdot 004$ \\
\hline
\end{tabular}

${ }^{\star}$ Smokers versus 'never' smokers.

$\dagger$ Positive results were defined by IgM titres $\geqslant 1: 16$ or a fourfold increase in IgG and/or IgM titres. 
pneumoniae and B. pertussis were found in three patients but no dual infections with $M$. pneumoniae and $C$. pneumoniae or M. pneumoniae and B. pertussis were observed.

All culture-, PCR- and/or serology-positive patients with $C$. pneumoniae and $M$. pneumoniae were treated with macrolides or fluoroquinolones for 1-2 weeks. Antibiotic treatment of adult patients infected with $B$. pertussis is controversial; erythromycin is the drug of choice but, unless it is administered early, antibiotic treatment does not alter the course of the disease (Linnemann \& Nasenbeny, 1977). Therefore, we treated about $30 \%$ of our patients empirically with antibiotics. After eradication of $C$. pneumoniae from the nasopharynx, the cough disappeared in all patients. Of these patients, however, three were still positive for $C$. pneumoniae by culture and/or PCR after 2 weeks of therapy. The results of laboratory tests and treatment of these patients with persistent cough are summarized in Table 2. One patient (no. 1) was treated with clarithromycin for 2 weeks but symptoms continued and $C$. pneumoniae was still detected by both culture and PCR. After 6 weeks of therapy with clarithromycin and inhaled steroid, symptoms disappeared completely and C. pneumoniae was no longer detected by PCR. The other two patients (nos 2 and 3 ) were also treated with clarithromycin for 2 weeks and their symptoms improved. However, their PCR results for C. pneumoniae were still positive. At 1 or 2 weeks after antibiotic treatment was discontinued, these patients again experienced a cough and visited our outpatient clinic. They were treated with clarithromycin and inhaled steroid for 3-4 weeks. Finally, their symptoms disappeared completely and the PCR results became negative for $C$. pneumoniae.

\section{DISCUSSION}

Respiratory tract infection is one of the most common causes of persistent cough and post-infectious cough is often persistent (Poe et al., 1989). Among respiratory pathogens, $B$. pertussis and M. pneumoniae are well-known causes of persistent cough in both children and/or adults (Wright $e t$ al., 1995; Hallander et al., 1999). Recently, some reports have indicated that $C$. pneumoniae infection may also cause persistent cough (Wright et al., 1997; Hallander et al., 1999; Kaneko et al., 1999). Wright et al. (1997) investigated the prevalence of acute C. pneumoniae infection in patients presenting with persistent cough for the first time. It was found that about $20 \%$ of patients with a cough lasting in excess of 2 weeks had serological evidence of an acute $C$. pneumoniae infection and concluded that $C$. pneumoniae infection may be responsible for a substantial proportion of prolonged coughing illnesses. However, their diagnostic methodology for C. pneumoniae was based on serology alone. Subsequently, Hallander et al. (1999) reported that C. pneumoniae was detected by PCR in 19 of 115 (17\%) children with coughs lasting less than 100 days. Kaneko et al. (1999) also detected C. pneumoniae by indirect immunofluorescence tests in 8 of 21 (38\%) children with persistent coughs. In contrast, Birkebæk et al. (2000) identified C. pneumoniae infection by PCR in only 3 of 201 adult patients with chronic coughs. In our study, we were able to detect $C$. pneumoniae infection by serology in 24 patients $(6.5 \%)$ and by culturing and/or PCR in $20(5.5 \%)$ of 366 adult patients with persistent coughs. It has been suggested that the serological criteria for the definition of an acute C. pneumoniae infection by the MIF test using serum is an IgG antibody titre of $\geqslant 1: 512$. This is a controversial issue because of the

Table 2. Laboratory findings in three patients with persistent coughs attributed to persistent C. pneumoniae infection

\begin{tabular}{|c|c|c|c|c|c|c|c|c|}
\hline \multirow{3}{*}{$\begin{array}{l}\text { Patient } \\
\text { no. }\end{array}$} & \multirow{3}{*}{$\begin{array}{c}\text { Age } \\
\text { (years) }\end{array}$} & \multirow[t]{3}{*}{ Sex } & \multirow{3}{*}{$\begin{array}{l}\text { Follow-up } \\
\text { (days after } \\
\text { onset of } \\
\text { symptoms) }\end{array}$} & \multirow{2}{*}{\multicolumn{2}{|c|}{$\begin{array}{l}\text { C. pneumoniae } \\
\text { detection }\end{array}$}} & \multicolumn{2}{|c|}{ Antibody titre } & \multirow{3}{*}{$\begin{array}{l}\text { Duration of } \\
\text { clarithromycin } \\
\text { treatment } \\
\text { (weeks) }\end{array}$} \\
\hline & & & & & & & & \\
\hline & & & & PCR & Culture & & & \\
\hline \multirow[t]{5}{*}{1} & 19 & M & 14 & + & + & 128 & 32 & 2 \\
\hline & & & 28 & + & + & 16 & 1024 & 2 \\
\hline & & & 42 & + & - & $<16$ & 1024 & 2 \\
\hline & & & 63 & - & - & $<16$ & 1024 & NA \\
\hline & & & 105 & - & - & $<16$ & 512 & NA \\
\hline \multirow[t]{4}{*}{2} & 22 & $\mathrm{~F}$ & 15 & + & - & 64 & 32 & 2 \\
\hline & & & 36 & + & - & $<16$ & 32 & 3 \\
\hline & & & 61 & - & - & $<16$ & 32 & NA \\
\hline & & & 95 & - & - & $<16$ & 32 & NA \\
\hline \multirow[t]{5}{*}{3} & 27 & M & 25 & + & - & $<16$ & 512 & 2 \\
\hline & & & 49 & + & - & $<16$ & 512 & 2 \\
\hline & & & 63 & + & - & $<16$ & 512 & 2 \\
\hline & & & 84 & - & - & $<16$ & 512 & NA \\
\hline & & & 111 & - & - & $<16$ & 512 & NA \\
\hline
\end{tabular}

NA, Not applicable. 
high incidence of IgG antibodies (at titres $\geqslant 1: 512$ ) seen among healthy asymptomatic subjects (Dowell et al., 2001). We also made the same observation (Miyashita et al., 2001c). In this study, we excluded IgG titres $\geqslant 1: 512$ from our diagnostic criteria. If Wright et al. (1997) had not used the positive serological criteria of IgG titres $\geqslant 1: 512,9 \%$ ( 6 of 65 subjects) of subjects would have met positive serology criteria for acute C. pneumoniae infection by the MIF test. This prevalence rate is almost the same as our serological results. However, the prevalence in this study, as judged by PCR, was higher than that reported by Birkebæk et al. (2000) but much lower than that reported by Hallander et al. (1999). This difference may be due to the use of a different cohort from a different geographical area than that employed in their study.

The rate of infection with $M$. pneumoniae was very low among our patients and similar to other reports (Wright et al., 1995; Birkebæk et al., 2000). These data, together with our data, indicate that $M$. pneumoniae infection may not cause persistent cough in adults. However, several studies have demonstrated the importance of B. pertussis as the cause of persistent cough among adults (Wright et al., 1995; Robertson et al., 1987; Nennig et al., 1996; Birkebæk et al., 1999). These authors reported a prevalence of B. pertussis infection of $12-26 \%$ in adult patients. In this study, we detected B. pertussis by serology in 68 patients $(18.5 \%)$ and confirmed that $B$. pertussis is a common cause of persistent cough in adults and should be considered in differential diagnosis.

C. pneumoniae is susceptible both in vitro and in vivo to macrolides, tetracyclines, ketolides and fluoroquinolones (Miyashita et al., 1997, 2001a, b). However, previous reports have noted that treatment failures are common and have suggested that persistent infection may be common after a conventional course of antibiotics (Hammerschlag et al., 1992; Miyashita et al., 1996a, 2002; Ekman et al., 1993; Falck et al., 1996, 1997; Normann et al., 1998; Block et al., 1995; Roblin \& Hammerschlag, 1998; Hammerschlag \& Roblin, 2000 a b). We have also found two cases of persistent $C$. pneumoniae infection after treatment with the appropriate antibiotics against C. pneumoniae pneumonia (Soda et al., 1997). In these two cases, we could not eradicate $C$. pneumoniae from the nasopharynx with a $2-3$ week course of treatment with macrolide or tetracycline and symptoms recurred. Furthermore, we have also seen persistent $C$. pneumoniae infection for up to 2 years in patients with diffuse panbronchiolitis who were asymptomatic most of the time but who also had exacerbations (Miyashita et al., 1996a).

It has been reported that there is an occurrence of continuing symptoms, relapsing illness or the development of complications with persistent infection due to $C$. pneumoniae (Hammerschlag et al., 1992; Miyashita et al., 1996a; Ekman et al., 1993; Falck et al., 1996). Anecdotal data suggest that prolonged therapy (i.e. greater than 2 weeks) may be desirable, since recrudescent symptoms have been described following erythromycin treatment for 2 weeks and even after
30 days of tetracycline or doxycycline treatment (Grayston et al., 1990; Kuo et al., 1995). Hammerschlag et al. (1992) reported refractory asthma in a patient with persistent $C$. pneumoniae infection, which was confirmed by culture, whose symptoms finally resolved after prolonged treatment with erythromycin. Furthermore, Ekman et al. (1993) reported that 9 of 20 patients with $C$. pneumoniae primary infection required more than one course of antibiotic therapy with erythromycin (7-14 days), doxycycline (1014 days) or tetracycline to improve their symptoms (four patients required three courses of treatment and five patients required two courses of treatment). In contrast, Falck et al. (1996) reported that they were not able to eradicate $C$. pneumoniae from the nasopharynx, despite several prolonged courses of antibiotics known to be effective against Chlamydia species. In the present study, follow-up cultures, PCR specimens and serology results were obtained from all patients who were positive by culturing and/or PCR. In three patients, we could not eradicate C. pneumoniae from the nasopharynx after 2 weeks of treatment with macrolide and symptoms either continued or relapsed. Therefore, we tried prolonged macrolide therapy consisting of 5-6 weeks of administration of clarithromycin to eradicate C. pneumoniae or to improve symptoms. Finally, we were able to eradicate $C$. pneumoniae from the nasopharynx. When C. pneumoniae could no longer be detected by culture or PCR, respiratory symptoms of these patients disappeared completely and no relapse was observed. However, we were not able to assess whether eradication of $C$. pneumoniae after anti-chlamydial therapy resulted in an improvement of their cough because patients were treated simulateously with steroid inhalation. Currently, data available indicate that the optimum dose and duration of therapy for persistent $C$. pneumoniae infection are uncertain. Therefore, new treatment strategies may be necessary to eradicate the organism in patients prone to persistent infection.

In conclusion, our data indicate that $C$. pneumoniae infection may cause persistent cough in adults. Furthermore, our data also indicate that it may be necessary to eradicate the organism when C. pneumoniae is detected by culture and/ or PCR in patients with persistent cough. More treatment studies with prolonged follow-up investigations of culture and/or PCR are needed to define more effective treatment strategies.

\section{ACKNOWLEDGEMENTS}

This work was supported by Project Research Grants from Kawasaki Medical School (13-401, 14-402).

\section{REFERENCES}

Birkebæk, N. H., Kristiansen, M., Seefeldt, T., Degn, J., Moller, A. Heron, I., Andersen, P. L., Moller, J. K. \& Ostergard, L. (1999). Bordetella pertussis infection and chronic cough in adults. Clin Infect Dis $\mathbf{2 9}$, $1239-1242$.

Birkebæk, N. H., Jensen, J. S., Seefeldt, T., Degn, J., Huniche, B., 
Andersen, P. L. \& Ostergard, L. (2000). Chlamydia pneumoniae infection in adults with chronic cough compared with healthy blood donors. Eur Respir J 16, 108-111.

Block, S., Hedrick, J., Hammerschlag, M. R., Cassell, G. H. \& Craft, J. C. (1995). Mycoplasma pneumoniae and Chlamydia pneumoniae in pediatric community-acquired pneumonia: comparative efficacy and safety of clarithromycin vs. erythromycin ethylsuccinate. Pediatr Infect Dis J 14, 471-477.

Dowell, S. F., Peeling, R. W., Boman, J. \& 11 other authors (2001). Standardizing Chlamydia pneumoniae assays: recommendations from the Centers for Disease Control and Prevention (USA) and the Laboratory Centre for Disease Control (Canada). Clin Infect Dis 33, 492-503.

Ekman, M. R., Grayston, J. T., Visakorpi, R., Kleemola, M., Kuo, C. C. \& Saikku, P. (1993). An epidemic of infections due to Chlamydia pneumoniae in military conscripts. Clin Infect Dis 17, 420-425.

Falck, G., Gnarpe, J. \& Gnarpe, H. (1996). Persistent Chlamydia pneumoniae infection in a Swedish family. Scand J Infect Dis 28, 271-273.

Falck, G., Gnarpe, J. \& Gnarpe, H. (1997). Prevalence of Chlamydia pneumoniae in healthy children and in children with respiratory tract infections. Pediatr Infect Dis J 16, 549-554.

Grayston, J. T., Campbell, L. A., Kuo, C. C., Mordhorst, C. H., Saikku, P., Thom, D. H. \& Wang, S. P. (1990). A new respiratory tract pathogen: Chlamydia pneumoniae strain TWAR. J Infect Dis 161, 618-625.

Hallander, H. O., Gnarpe, J., Gnarpe, H. \& Olin, P. (1999). Bordetella pertussis, Bordetella parapertussis, Mycoplasma pneumoniae, Chlamydia pneumoniae and persistent cough in children. Scand J Infect Dis 31, 281-286.

Hammerschlag, M. R. \& Roblin, P. M. (2000a). Microbiologic efficacy of moxifloxacin for the treatment of community-acquired pneumonia due to Chlamydia pneumoniae. Int J Antimicrob Agents 15, 149-152.

Hammerschlag, M. R. \& Roblin, P. M. (2000b). Microbiological efficacy of levofloxacin for treatment of community-acquired pneumonia due to Chlamydia pneumoniae. Antimicrob Agents Chemother 44, 1409.

Hammerschlag, M. R., Chirgwin, K., Roblin, P. M., Gelling, M., Dumornay, W., Mandel, L. \& Smith, P. (1992). Persistent infection with Chlamydia pneumoniae following acute respiratory illness. Clin Infect Dis 14, 178-182.

Kaneko, K., Yamashiro, Y., Maruyama, T. \& Obinata, K. (1999). Chlamydia pneumoniae infection in children with persistent cough. Arch Dis Child 80, 581-582.

Kuo, C. C., Jackson, L. A., Campbell, L. A. \& Grayston, J. T. (1995). Chlamydia pneumoniae (TWAR). Clin Microbiol Rev 8, 451-461.

Linnemann, C. C., Jr \& Nasenbeny, J. (1977). Pertussis in the adult. Annu Rev Med 28, 179-185.

Miyashita, N., Kubota, Y., Kimura, M., Nakajima, M., Niki, Y., Soejima, R. \& Matsumoto, A. (1994). Characterization of a Chlamydia pneumoniae strain isolated from a 57-year-old man. Microbiol Immunol 38, 857-864.

Miyashita, N., Matsumoto, A., Kubota, Y., Nakajima, M., Niki, Y. \&
Matsushima, T. (1996a). Continuous isolation and characterization of Chlamydia pneumoniae from a patient with diffuse panbronchiolitis. Microbiol Immunol 40, 547-552.

Miyashita, N., Matsumoto, A., Soejima, R., Kubota, Y., Kishimoto, T., Nakajima, M., Niki, Y. \& Matsushima, T. (1996b). Evaluation of a direct fluorescent antibody assay for detection of Chlamydia pneumoniae. Jpn J Assoc Infect Dis 70, 224-231.

Miyashita, N., Niki, Y., Kishimoto, T., Nakajima, M. \& Matsushima, T. (1997). In vitro and in vivo activities of AM-1155, a new fluoroquinolone, against Chlamydia spp. Antimicrob Agents Chemother 41, 1331-1334.

Miyashita, N., Fukano, H., Niki, Y. \& Matsushima, T. (2001a). In vitro activity of telithromycin, a new ketolide, against Chlamydia pneumoniae. J Antimicrob Chemother 48, 403-405.

Miyashita, N., Niki, Y. \& Matsushima, T. (2001b). In vitro and in vivo activities of sitafloxacin against Chlamydia spp. Antimicrob Agents Chemother 45, 3270-3272.

Miyashita, N., Niki, Y., Nakajima, M., Fukano, H. \& Matsushima, T. (2001c). Prevalence of asymptomatic infection with Chlamydia pneumoniae in subjectively healthy adults. Chest 119, 1416-1419.

Miyashita, N., Fukano, H., Hara, H., Yoshida, K., Niki, Y. \& Matsushima, T. (2002). Recurrent pneumonia due to persistent Chlamydia pneumoniae infection. Intern Med 41, 30-33.

Nennig, M. E., Shinefield, H. R., Edwards, K. M., Black, S. B. \& Fireman, B. H. (1996). Prevalence and incidence of adult pertussis in an urban population. JAMA 275, 1672-1674.

Normann, E., Gnarpe, J., Gnarpe, H. \& Wettergren, B. (1998). Chlamydia pneumoniae in children with acute respiratory tract infections. Acta Paediatr 87, 23-27.

Poe, R. H., Harder, R. V., Israel, R. H. \& Kallay, M. C. (1989). Chronic persistent cough. Experience in diagnosis and outcome using an anatomic diagnostic protocol. Chest 95, 723-728.

Robertson, P. W., Goldberg, H., Jarvie, B. H., Smith, D. D. \& Whybin, L. R. (1987). Bordetella pertussis infection: a cause of persistent cough in adults. Med J Aust 146, 522-525.

Roblin, P. M. \& Hammerschlag, M. R. (1998). Microbiologic efficacy of azithromycin and susceptibilities to azithromycin of isolates of Chlamydia pneumoniae from adults and children with community-acquired pneumonia. Antimicrob Agents Chemother 42, 194-196.

Soda, K., Kishimoto, T., Kubota, Y., Matsushima, T., Izutsu, H., Matsumoto, A. \& Soejima, R. (1997). An outbreak of Chlamydia pneumoniae in a junior high school and its district: presence of asymptomatic oropharyngeal carriers in healthy subjects and in cases of pneumonia after a clinical cure. Amakusa Med J 11, 17-28 (in Japanese).

Wright, S. W., Edwards, K. M., Decker, M. D. \& Zeldin, M. H. (1995). Pertussis infection in adults with a persistent cough. JAMA 273, 1044-1046.

Wright, S. W., Edwards, K. M., Decker, M. D., Grayston, J. T. \& Wang, S. P. (1997). Prevalence of positive serology for acute Chlamydia pneumoniae infection in emergency department patients with persistent cough. Acad Emerg Med 4, 179-183. 